\title{
Design of Optical Fiber SPR Sensing System for Water Quality Monitoring
}

\author{
Xuehua Jiang ${ }^{1, a}$ and Qingmin Meng ${ }^{1, b}$ \\ ${ }^{1}$ School of Automobile, Linyi University, Shandong, 276000, China \\ ajxhyx@163.com, b mengqingmin@lyu.edu.cn
} Keywords: Water quality monitoring, Surface plasmon resonance, Sensing systems, Detection
performance

\begin{abstract}
Water quality monitoring was a basic work for water quality resource management, there were some problems in the current detection means for oily waste water, such as low measurement accuracy, off line detection, an so on. Based on the principle of surface plasmon resonance (SPR), a optical fiber sensor was designed, which could monitor the interaction of biological molecules online and real-time, with label-free, without separation and purification. It was a non-contact detection technology and it was easy to implement remote monitoring. The designed optical fiber SPR sensor system was tested through oily waste water with different concentrations. The experimental results showed that the optical fiber SPR sensing system could measure the oil in waste water with high accuracy, fast speed, and good stability, the method achieved on-line monitoring and improved the real-time of detection and the ease of operation.
\end{abstract}

\section{Introduction}

Since the 1970s, with the development of optical fiber technology and communication technology, the optical fiber sensing technology has been a new type of sensor technology, representing the development trend of the new generation of sensors. The biological sensors about water quality testing have been researched, which can be used for environmental monitoring. Most of them are expensive and unstable, the main reason lies in the biological signals transformation.

Water quality monitoring is a basic work for water resource management. The currently used detection methods are not conducive to online or on-site inspection, it is difficult to apply to the actual operation of the water quality detection system. The optical fiber sensing signal based on surface plasmon resonance (SPR) is less susceptible to external factors, such as mechanical structure, light fluctuations, and so on. It has several advantages of label free, high selectivity, high speed responsibility and lower sample consumption, then the technology is an important way to detect pollutant $[1,2]$.

\section{Total structure of sensing system}

The water quality monitoring system based on SPR uses the physical optics theory of surface plasma resonance effect and utilizes the relationship between medium refraction index and resonance wavelength, and obtains the refraction index by measuring the light intensity and resonance wavelength $[3,4]$. Applying the SPR detection technology to test water quality is a multi-disciplinary research.

An optical fiber surface plasmon sensor system is designed based on resonance light intensity modulation technology. The required performance indicators are as follows:

(1) Temperature range: $0-100^{\circ} \mathrm{C}$;

(2) Refraction index detection range: $1.3-1.7$;

(3) Testing sensitivity of refraction index: $<10-5$;

(4) SNR: 250dB;

(5) Error of repeatability and stability: $<0.1 \%$. 
The system mainly includes three parts: sensing part, data acquisition part and computer processing part, the overall structure is given, as shown in Fig. 1.

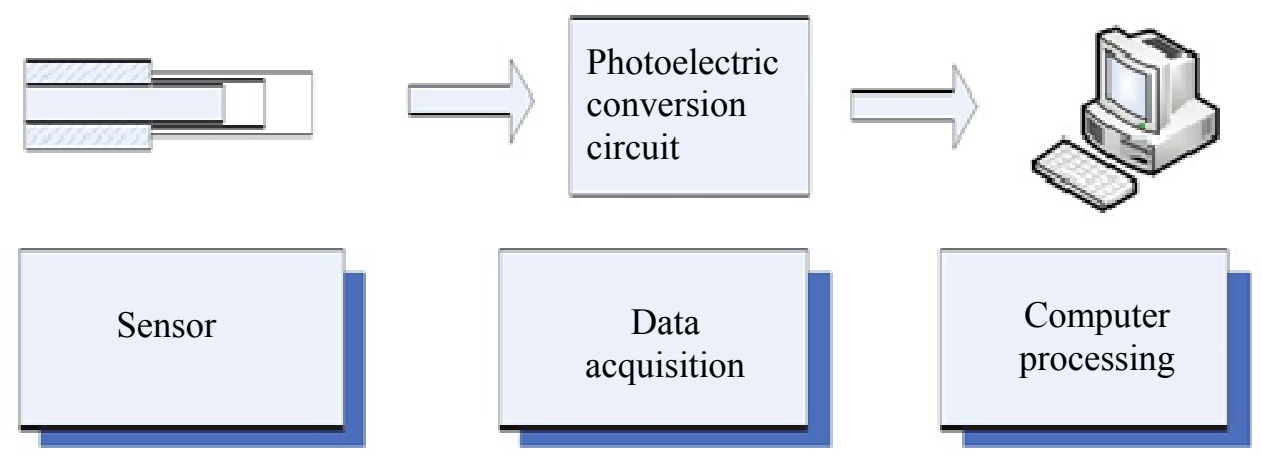

Fig. 1 The whole module structure of detection system

The sensing part is very key in the design of detection system, which working principle is SPR intensity modulation. Considering the several factors affecting sensor performance [5,6], it is optimized in the following four areas. (1) Combining the optical optical and SPR principle together and making the optical fiber as coupling device, the light-wave is coupled with SPR to overcome the shortcomings of traditional absorbance detection methods. (2) Using the SPR sensor probe with two-layer coating optical fiber and coupling Y-type optical system improves the sensitivity and stability of sensor response. (3) The white light source with high stability is used to make the sensor mark free and separation free. (4) The noise characteristic of optical fiber sensor is analyze, and the de-noising methods and structure are optimized to improve the detection accuracy, timeliness and operation convenience.

The data acquisition part is composed of a coaxial probe YSPD618 and filter amplifier circuit. The data acquisition card DAQCard6024E collects data which can be displayed on the computer.

The computer processing part uses Labview programming to analyze and process the collected data, save and display the measurement results.

\section{Manufacture of sensing system}

\section{Design and manufacture of sensor}

The manufacture of the optical fiber SPR sensor comprises several technological processes, such as cutting, polishing, cleaning, coating, and on on, as shown in Fig. 2.

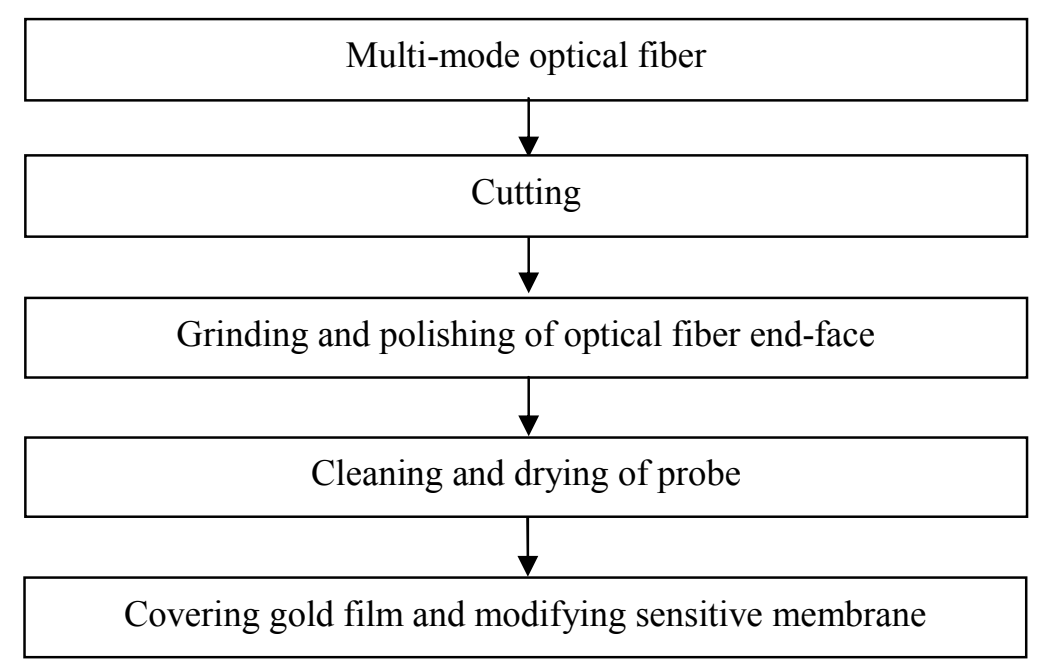

Fig. 2 Preparation process of optical fiber SPR sensor 
In this project, the multi-mode step-index silica fiber is used, which core diameter is $600 \mu \mathrm{m}$ and the core refraction index is $1.458 \mathrm{RIU}$. The dedicated optical fiber cleaver is used to cut and grind optical fiber facing, so that the end is perpendicular to the fiber meridional plane and to ensure that the reflection angles are equal when light back and forth. The etching method can remove the cladding part of core surface, then the ultrasonic method can wash out the residue and dirt exposed in surface. At the same time, the gold is selected to make as the basement, and $\mathrm{Cr}$ or $\mathrm{Ti}$ is plated to increase the adhesion of gold film.

The light transmitting principle of optical fiber SPR sensor is shown as Fig. 3, where, $D$ represents the core diameter, $d_{1}$ is the thickness of gold film, $L$ represents the length of sensing area, $\theta_{i n}$ indicates the coupling angle of incident light, $\theta$ is the incidence angle of SPR effect, $\varepsilon_{0}, \varepsilon_{1}$ and $\varepsilon_{2}$ are permittivity of core, gold film and surrounding medium respectively. The sensor designed in this project is given, as shown in Fig. 4.

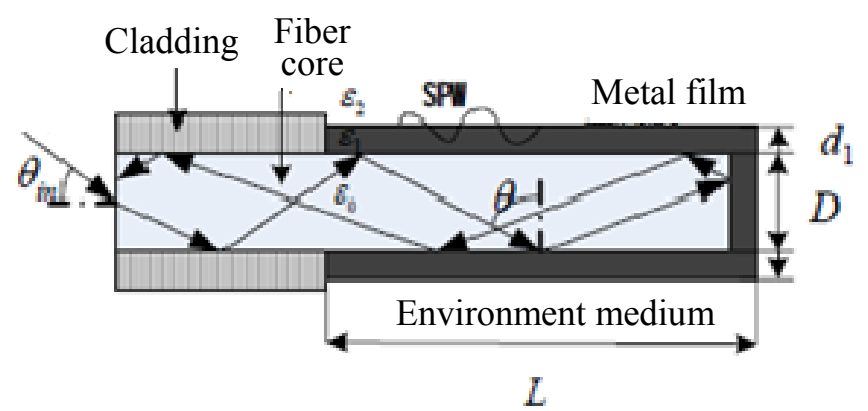

Fig. 3 Light transmitting principle of optical fiber SPR sensor

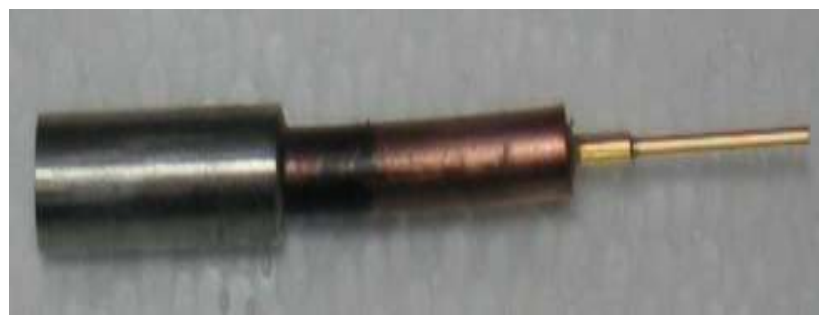

Fig. 4 Physical photo of optical fiber SPR sensor

\section{Design and manufacture of light source}

Light source is an important part in the sensing system, which can determine whether the optical fiber system can achieve the expected characteristics or not. There are many sources that optical fiber sensors can use. According to the coherence of light, they can be divided into coherent light source and non-coherent light source.

The optical fiber SPR sensing System uses a white light source, requiring that the light can change continuously from $300 \mathrm{~nm}$ to $800 \mathrm{~nm}$ and it is continuous, smooth and there is no mutation in this band. Then, this project selects the tungsten halogen lamp as the light source. Due to the requirements of high stability for light source, it is necessary to adopt a highly stable power supply, then, a self designed highly stable DC power source is adopted in this testing system.

\section{Design and manufacture of optical fiber coupler}

Since it should analyze the light spectral of output signal in this project, then what the system really cares about is not the absolute intensity. It can use the Y-type optical fiber coupler. The light couples into one end of the Y-type optical fiber through lens, goes into optical fiber sensors by optical interface, and then spreads into the spectrometer by the reflection of optical fiber sensor head. The structure of Y-type fiber optic coupler is given, as shown in Fig. 5. The incident and emergent fibers are composed of multi-mode optical fibers with the core diameter of $600 \mu \mathrm{m}$. Their incoming and 
exiting ends all use SMA905 interfaces, and then they are connected at the other ends. The Y-type optical fiber coupler designed in this project is given, as shown in Fig. 6.

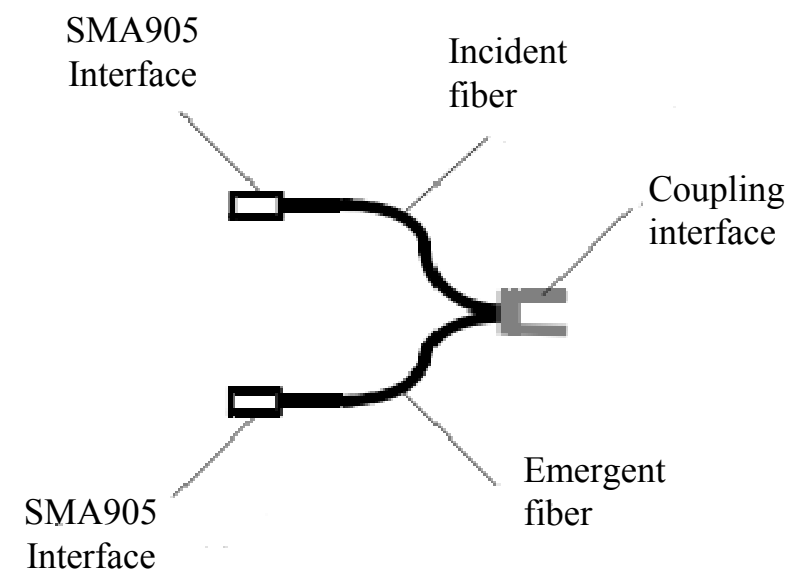

Fig. 5 Structure of Y-type optical fiber coupler

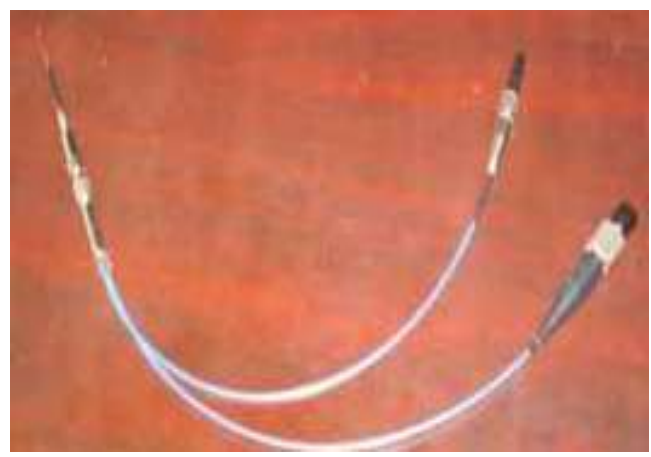

Fig. 6 Physical photo of Y-type optical fiber coupler

\section{Performance test of sensing system}

The optical fiber SPR sensor is used to test glycerine solution with different solubilities. The spectrum analyzer CX-9800 (T) is used to compare the reults of same aqueous solution, and then the sensitivity of refraction index, signal to noise ratio, repeatability and stability are tested [7]. The detection results show that the system performance can meet the design requirements.

(1) Sensitivity of refraction index

The probe of fiber optic SPR sensor is put into the glycerine solutions with different solubilities. The refraction index of optical fiber SPR sensor is measured, and its range is 1.3-1.7, and the sensitivity is $9.467 \times 10^{-6}$.

(2) Signal to noise ratio

According to the test data, the signal to noise ratio of optical fiber SPR sensor is measured which is 249-252dB.

(3) Repeatability and stability

The spectrum analyzer is used to test the transmitted spectra. When the system is tested many times, the stability error is less than $0.09 \%$ which can meet the design indicators.

\section{Conclusions}

This project designs and realizes a optical fiber SPR sensor, which is used to measure the oil in waste water.

(1) On the basis of analyzing the theory of surface plasmon resonance, combining optical fiber and SPR principle together, making the optical fiber as the coupling device, the light waves and the 
surface plasmons are coupled and resonated. Then, it makes sensing mark free and seperate free. The method can achieve online monitoring and improve the real-time of testing and convenience of operation.

(2) The system adopts SPR sensor probe with two-layer coated optical fiber and coupling Y-type optical fiber system to improve the detection performance of fiber optic SPR sensing system. It has many advantages, such as not subject to electromagnetic field, non-contact detection, high response speed, and so on. It can improve the sensitivity, anti-interference performance, and it has high accuracy, speed and good stability.

3. The designed optical fiber SPR sensor is small in size and suitable for remote monitoring. The system can test many indicators at the same time, including water pollution concentration, water temperature, turbidity, and so on. Then, it has an important application in the field of environmental monitoring and protection, industrial safety, and it can produce good economic and social benefits.

\section{Acknowledgement}

This study is supported by the Science and Technology Development Project of Shandong Province, China (No. 2011YD010066). The authors acknowledge the support of the Department of Science \& Technology of Shandong Province and Linyi University.

\section{References}

[1] X. Jiang, Q. Meng, M, Zhang, Study and Experiment of Fiber Sensor based on SPR. Transducer and Microsystem Technologies. 28(7) (2009) 33-36.

[2] State Environmental Protection Administration, Water and Waster Water Monitoring Method. China Environmental Science Press, Beijing, China, (2002).

[3] N. Yoshida, S. J. McNiven, A. Yoshida, T. Morita, H. Nakamura, I. Karube, A compact optical system for multi-determination of biochemical oxygen demand using disposable strips. Field analytical chemistry and technology. 5(5) (2001) 222-227.

[4] R.L. Meyer, T. Kjaer,N.P. Revsbech, Use of NOx-microsensors to Estimate the Activity of Sediment Nitrification and NOx- consumption along an Estuarine Salinity, Nitrate, and Light Gradient. Aquatic microbial ecology. 26(2) (2001) 181-193.

[5] Z. Zhang, W.Yin, T. Wen, L. Zhu, J. Zhang, Study on the relation expression of solution concentration and its refractive index.Journal of north university of China(Natural science edition). 30(3) (2009) 281-285.

[6] Q. Meng, Influencing Factors of Optical Fiber SPR Sensor Performance. Transducer and Microsystem Technologies. 29(5) (2010) 47-49.

[7] X. Jiang, Q. Meng, Online Measurement of Oil in Waste Water Based on Surface Plasmon Resonance Fiber Sensor. Advanced Materials Research. 850-851 (2014) 1372-1375. 